\title{
Les propriétés collectives face aux attaques
} libérales (1750-1914). Europe occidentale et Amérique latine

\section{Guy Lemarchand}

\section{(2) OpenEdition \\ Journals}

Édition électronique

URL : https://journals.openedition.org/ahrf/1867

DOI : 10.4000/ahrf.1867

ISSN : 1952-403X

Éditeur :

Armand Colin, Société des études robespierristes

Édition imprimée

Date de publication : 1 décembre 2004

Pagination : 177-180

ISSN : 0003-4436

\section{Référence électronique}

Guy Lemarchand, «Les propriétés collectives face aux attaques libérales (1750-1914). Europe occidentale et Amérique latine ", Annales historiques de la Révolution française [En ligne], 338 | octobredécembre 2004, mis en ligne le 22 mars 2006, consulté le 24 avril 2022. URL : http:// journals.openedition.org/ahrf/1867 ; DOI : https://doi.org/10.4000/ahrf.1867

Ce document a été généré automatiquement le 24 avril 2022.

Tous droits réservés 


\title{
Les propriétés collectives face aux attaques libérales (1750-1914). Europe occidentale et Amérique latine
}

\author{
Guy Lemarchand
}

\section{RÉFÉRENCE}

Marie-Danielle Demélas et Nadine Vivier (dir.), Les propriétés collectives face aux attaques libérales (1750-1914). Europe occidentale et Amérique latine, Rennes, Presses Universitaires de Rennes, 2003, 330 p., ISBN 2-86847-872-7, $22 €$.

1 C'est un livre particulièrement utile et neuf que présentent les deux maîtresses d'œuvre de cette entreprise collective. Elles ont en effet tenté un genre peu répandu dans l'historiographie française, l'histoire comparée internationale. Et pour ce faire les obstacles étaient importants. Il y a d'abord le flou du vocabulaire désignant les terres d'usage communautaire, aussi bien dans les sources que dans les travaux des historiens. Il en résulte évidemment une difficulté de traduction en vue d'une comparaison entre les pays. À cet égard est essentielle la distinction, banale pour les Français, mais beaucoup moins nette en Angleterre ou en Espagne entre droits agraires collectifs (vaine pâture, parcours, etc.), biens patrimoniaux communautaires (four banal municipal en Provence par exemple) et fonds de propriété collective. De même s'impose de différencier terres boisées et étendues en landes bonnes surtout au pâturage extensif. Une seconde difficulté tient au développement inégal des études d'une nation à l'autre : alors que la France, la Belgique et la Grande Bretagne sont bien pourvues à cet égard, l'Italie, l'Allemagne et bien plus encore l'Amérique latine souffrent d'un certain retard dans les recherches sur le sujet. Enfin le cadre même de ces travaux présente des avantages mais également des inconvénients sérieux. Aujourd'hui, et l'ouvrage est ainsi ordonné à juste titre, on procède à la présentation de 
la question État par État, ce qui met bien en valeur le rôle des gouvernements et des administrations dans l'évolution pluriséculaire de ces terres. De plus ce cadre s'impose à l'historien parce que la consultation des archives s'organise d'abord dans les dépôts d'archives nationaux où sont conservés enquêtes, lois et débats ordonnés par les monarchies depuis le XVIIIe siècle sur les bienfaits ou l'inutilité de ces espaces ruraux. Mais chaque État dans l'Ancien Régime en fait n'est pas homogène et les situations s'opposent d'une province à l'autre, particulièrement en Allemagne, en Italie, et même dans les États relativement centralisés, la France, l'Angleterre, le Portugal, ou dans les petites formations étatiques comme les Pays-Bas du Sud, la Suisse, et dans les vastes étendues à domination unique, l'Amérique espagnole. L'étendue des communaux varie beaucoup d'une province à l'autre, forte à plus de $30 \%$ du sol dans l'est de la France et le Jura, dans le Portugal du Nord-Est et le Norte Espagnol, mais faible à inexistante en Île-de-France et dans le Hainaut, au Sud de Lisbonne ou en Catalogne. De plus, le régime de propriété de ces terres et les modalités de leur jouissance ne sont pas non plus uniformes. Propriété éminente du landlord dans une grande partie de l'Angleterre, elles appartiennent aux communautés d'habitants ou aux familles les plus notables de celles-ci en Italie du Nord. Leur accès est loin aussi d'être ouvert à tous et même, avec la pression démographique et la mercantalisation de l'économie rurale, on tend à le restreindre de plus en plus : égal pour l'ensemble des paysans en Picardie et en Flandre, il est limité selon le nombre des bêtes possédées en Béarn, tandis que le droit de jouissance peut se vendre indépendamment des biens-fonds dans le Plateau suisse. Sans dissimuler les faiblesses de notre connaissance des pays recensés, les deux directrices ont néanmoins dressé une typologie qui aidera fortement à orienter les discussions futures, même si on peut diverger sur tel ou tel point comme par exemple mettre dans le même groupe l'Angleterre et la partie orientale du royaume de Prusse, région de deuxième servage à fort pouvoir seigneurial.

2 Avec une chronologie qui est propre à chaque État, on retrouve en gros la même évolution d'un pays à l'autre en Europe et même en Amérique. C'est à partir du milieu du XVIIIe siècle que la question de l'utilité des terres collectives est posée publiquement, d'abord en Grande-Bretagne et en France, sous l'influence du libéralisme individualiste, de la physiocratie et de l'agronomie et sous la pression d'une croissance démographique qui s'accélère. Très vite le débat gagne à peu près toute l'Europe et les élites éclairées se prononcent clairement pour la privatisation de ces terres à faible rendement par mauvaise exploitation, dit-on, et qui entretiendraient la paresse chez les bénéficiaires, point de vue qui satisfait les appétits de terre des seigneurs dans certaines provinces de France comme au Portugal ou de la bourgeoisie en Italie du Nord, ou encore des hacendados au Mexique. Cette image péjorative a été partiellement adoptée par les historiens, quelle que soit leur orientation, jusqu'aux années 1980. Portée par les Lumières attachées à la propriété individuelle, la Révolution française donne à l'idée de privatisation un nouvel éclat, bien que les autorités révolutionnaires de notre pays aient été très prudentes et ambiguës. Au XIXe siècle, à mesure que devient plus vive la pression du libéralisme bourgeois et que s'accélère l'augmentation de la population et la faim de terres à blés, les États par la loi autorisent le partage général dont ne profitent pas nécessairement d'ailleurs, tant s'en faut, la paysannerie, particulièrement par exemple en Espagne. Pourtant là où elles sont fortement constituées et relativement homogènes socialement ou ethniquement, les communautés résistent à la marchandisation de la terre excitée par les cultures nouvelles et la construction des voies de circulation des marchandises, dans le monte de 
la Péninsule Ibérique et plus encore au Pérou et Bolivie, loin des grands axes commerciaux.

3 En Angleterre et au Pays de Galle les communaux sont régis par les cours manoriales. Ils sont particulièrement étendus dans le Nord, l'Ouest et le Sud-Ouest et en 1690 représentent encore peut-être 3,6 millions d'ha. C'est le Parlement qui leur a porté les coups les plus durs avec les actes privés ordonnant le partage à l'échelle d'une paroisse, sporadiquement d'abord au XVIIe siècle, puis en multipliant les décisions après 1720 et avec les Public general enclosure acts de 1801, 1836, 1840 et 1845, ce qui aboutit à l'expulsion de multiples cottagers et même des yeomen. Néanmoins J. Neeson escamote malheureusement la difficulté en passant à peu près sous silence l'important mouvement d'enclosure qui avait commencé bien avant 1700, chacun en effet se souvient de la plainte de Thomas More à propos de la fin du XVe siècle et du début du XVIe siècle. En France la question nous est bien connue avec les travaux récents de $\mathrm{N}$. Vivier qui résume ici sa thèse. Si les édits royaux de partage facultatif de 1789-1790 ont eu peu de succès, la loi du 10 juin 1793 a connu plus d'application. Puis les besoins financiers de l'Empire en 1813-1815 ont poussé à la mise en vente des communaux et à partir de la Monarchie de Juillet les préfets reprennent cette politique, tandis que le code forestier de 1827 exclut de fait nombre d'usagers des lois. En 1846, la propriété commune ne couvre plus que $9 \%$ du territoire.

En Belgique, les landes et surtout les forêts communes ne sont étendues, d'après le recensement de 1846, qu'en Limbourg et Luxembourg. En fait, l'attaque contre les communaux a commencé dès le XVIe siècle à l'initiative des États Provinciaux et également de seigneurs, bourgeois et paysans aisés usurpant le territoire commun. Ce mouvement s'accélère sous Marie-Thérèse et Joseph II hostiles aux usages collectifs. Au Portugal, la monarchie, par souci fiscal et recherche de la stabilité générale, chercha longtemps à protéger la propriété communale des baldios dans le code de 1603 qui les reconnaît officiellement et la loi de 1822 qui les déclare propriété du peuple. Néanmoins, l'Académie des Sciences créée en 1779 préconise leur suppression et la loi de 1869 les désamortit. En Espagne ou comme au Portugal de vastes étendues ont été concédées par les fueros aux communautés lors de la Reconquista, et il y a en encore 185023 \% de la surface qui est en usage communautaire. Mais ne sont vraiment collectif que les baldios ouverts au plus grand nombre, alors que les proprios sont affermés pour subvenir aux besoins municipaux, et peu à peu les seconds s'étendent aux dépens des premiers à l'incitation des notables de villages. Sous Charles III les Lumières trouvent un certain écho et le roi ordonne des privatisations de baldios à 5 reprises, en vue aussi d'accroître l'impôt sur les municipalités. Et ce même souci fiscal pousse à des ventes de communaux par des levées extraordinaires en 1780-1783, 1792-1793, 1800 et 1813. Évidemment les Cortès de Cadix s'affirment en 1813 hostiles aux terres collectives, mais c'est seulement en 1855 qu'est promulguée la possibilité de les aliéner toutes, tandis qu'avait déjà été créée une administration forestière en 1833-1836 qui limite l'utilisation des forêts. En Allemagne s'oppose la région du Sud-Ouest à fortes communautés et élevage important en dépit de surfaces en pâture réduites, au Nord où elles sont faibles mais disposent de vastes marais et landes, le partage s'est fait au XIXe siècle sous l'impulsion des États. En Italie on retrouve en Sardaigne, en Ligurie et comme en Vieille Castille espagnole, une pleine propriété des communautés couvrant tout le terroir avec redistribution périodique des lopins de culture, avec de vastes pâtures collectives, tandis que la Lombardie et la Vénétie en ont peu et que les propriétés individuelles y sont stables. Les premières mesures de partage local sous 
l'égide de l'État datent du despotisme éclairé en Toscane (1776-1778) et Lombardie (1770-1775) et, freinés par la pression paysanne, les pouvoirs publics ne prennent une mesure d'ensemble d'abolition des usages en forêt domaniale qu'avec l'unification en 1875. En Suisse, à l'ouest ouvert surtout, une première vague de partages a lieu dès le XVIe siècle puis dans la seconde moitié du XVIIIe et au XIXe siècle, soutenue par l'autorisation de l'administration de certains cantons où bourgeois et paysans aisés sont influents. Mais la propriété collective se maintient fortement pour les alpages.

5 Pour le statut des terres l'Amérique latine ressemble fort à la Péninsule Ibérique. Mais la conquête et la colonisation introduisent un élément original primordial: la séparation spontanée et voulue à la fois et l'exploitation d'une population vaincue par la minorité européenne. En effet les conquistadores obtiennent du roi d'Espagne de vastes propriétés et pour les travailler le système de l'encomienda organisé pour l'occasion leur fournira la main d'œuvre des villages indiens voisins qui leur sont assignés et qui doivent en outre le tribut au monarque. Comme le rappelle commodément E. Samudio, les ordonnances de 1518 proclament le respect des terres indigènes traditionnelles, ce qui est réédité indirectement en 1550 et 1560 . Toutefois, et cela offrira une couverture juridique aux Espagnols sur le terrain quelle que soit la volonté des souverains, dès 1546 Charles-Quint décide qu'un encomiendero pourra hériter des Indiens sans descendance et que les indigènes devraient être rassemblés en reductiones et en 1591-1598 est formulé le principe de composition des terres selon lequel les établissements espagnols doivent recevoir les fonds qui leur sont nécessaires pour vivre et qu'on peut prendre sur les terres indiennes à condition de leur redonner d'autres fonds suivant leur besoin. Et en fait dès le début de la conquête la dépossession des indigènes par héritage, achat et usurpation commence - j'ajouterai facilitée par la chute de la population originelle - d'autant plus aisément que la propriété indienne est indivise et collective, le resguardo dont ils ont l'usufruit. L'audiencia du lieu répartit celle-ci (repartimiento) et leur en donne l'usage, de façon à contrôler les hommes et assurer la mise en valeur des fonds des Espagnols et produire leur propre subsistance, ce qui implique des regroupements de communautés ou fractions de communautés indigènes. La monarchie s'efforce donc de mener une double politique de préservation des Indiens et de développement des propriétés espagnoles, mais sous Charles III et Charles IV elle penche de plus en plus pour le productivisme et favorise nettement les colons avec le souci de stimuler le commerce métropolitain et d'augmenter les recettes fiscales. L'idée de partager les terres communautaires entre les Indiens se fait jour afin d'accroître la production agricole, et en 1799 Godoy prend des mesures afin de faciliter l'aliénation de biens collectifs divers. Si les Cortès de Cadix maintiennent en 1813 l'inaliénabilité des terres indigènes des Indes Occidentales, les États nés ensuite de l'indépendance reprennent et accentuent cette politique libérale. M.-D. Demélas fournit d'ailleurs une table chronologique utile pour les cinq pays d'Amérique post-coloniale traités.

6 Au Mexique les États de la République fédérale commencent à répartir dans les années 1824-1835 les terres entre les pères de famille, puis la résistance indienne contribue à arrêter le mouvement qui reprend et va s'amplifier avec la loi de désamortissement de 1856 qui aboutit à la disparition d'une grande partie de la propriété communautaire. Au Guatemala où existe encore une forte population indigène, après avoir distribué les grands domaines d'Eglise en 1825-1838 et avoir voulu s'en prendre aux terres indiennes, ce qui provoque une rébellion rurale (1836), la politique libérale est à nouveau appliquée dans les années 1860, mais l'opposition demeure vive. Au 
Venezuela, c'est dès 1811 que la constitution de la première République impose la répartition des terres collectives, mais Bolivar décrète par souci de justice en 1821 la restitution des fonds confisqués aux Indiens tout en incitant au partage des resguardos et en 1882 la loi oblige à les diviser. Au Pérou les libérateurs confisquent les biens des partisans de l'Ancien Régime et répartissent sans ménagement les terres indigènes réputées excédentaires (1821-1826). Le manque de finances oblige même le nouvel État à rétablir le tribut des Indiens (1826-1854). Mais la population soumise qui augmente même, s'accroche à ses terres, utilisant entre autres les confréries religieuses à cette fin, et ce n'est qu'après 1870 que s'établit un marché foncier au profit partiellement des anciens encomendieros devenus propriétaires d'haciendas latifundiaires. En Bolivie il en va à peu près de même, la république cherche à démembrer dès 1824 les communautés indigènes, toutefois ce n'est qu'après la loi de 1863 que débute un désamortissement limité.

7 Comme toujours dans un ouvrage collectif les contributions n'atteignent pas toutes le même degré de précision et quelques unes restent assez confuses, faute de la même rigueur conceptuelle partout. Il faudrait aussi replacer davantage la propriété collective dans le cadre du système de culture et d'élevage en vigueur: perdre le communal dans une plaine de grande culture commerciale n'a pas la même importance sociale que dans une région accidentée à élevage prédominant d'échange restreint. De même, quoiqu'évoqué, le facteur démographique, densité de population, mouvement général et migrations, n'est pas toujours suffisamment pris en compte. Il est vrai qu'on ne le connaît pas toujours nettement, particulièrement en Amérique latine. Enfin, on peut s'étonner que n'aient guère été traités les Provinces-Unies ni les pays scandinaves, pour lesquels les travaux ne manquent pas. Cependant ces réserves limitées ne sauraient effacer le grand intérêt de ce livre. 